\title{
CrystEngComm
}

Check for updates

Cite this: CrystEngComm, 2022, 24, 609

Received 11th November 2021, Accepted 9th December 2021

DOI: 10.1039/d1ce01516h

rsc.li/crystengcomm

\section{The extensive solid-form landscape of sulfathiazole: geometrical similarity and interaction energies $\uparrow$}

\author{
David S. Hughes, (iD*ab Ann L. Bingham, ${ }^{\text {b }}$ Michael B. Hursthouse, ${ }^{b}$ \\ Terry L. Threlfall ${ }^{b}$ and Andrew D. Bond (iD)*a
}

A set of 96 crystal structures containing sulfathiazole (SLFZ) is presented, comprising 52 new crystal structures and 39 structures retrieved from the Cambridge Structural Database. The set comprises five polymorphs, 59 co-crystals, 29 salts and three other structures, providing one of the most extensive solidform landscapes established for a single active pharmaceutical ingredient. The crystal structures are energy-minimised using DFT-D calculations to yield a standardised set. Geometrical comparisons are made using the programs CrystalCMP, COMPACK and XPac, and the results are combined and compared. Consistent conclusions are drawn on full 3-D isostructurality within the set, identifying a group of 18 isostructural co-crystals, and 11 further isostructural groups of salts or co-crystals comprising two or three structures. Aside from the fully isostructural groups, common 2-D supramolecular constructs (SCs) are restricted to groups of only two or three structures and there are no 2-D SCs that are observed especially frequently. Transferable 1-D SCs are more common, and examples are identified based on hydrogenbonded and non-hydrogen bonded interactions between SLFZ molecules. Closely-related 1-D SCS comprising translated SLFZ molecules linked by hydrogen bonds are found in one polymorph and almost half of the multi-component set. A comparison of the five SLFZ polymorphs and the 91 multi-component crystal structures identifies several pairwise interactions between SLFZ molecules that are present in one of the polymorphs and at least one multi-component structure. A centrosymmetric $\mathrm{R}_{2}^{2}(8) \mathrm{N}-\mathrm{H} \cdots \mathrm{N}$ hydrogenbonded pair occurs in one polymorph and approximately $80 \%$ of the co-crystals. Intermolecular interaction energies, calculated using the PIXEL method, show that this $\mathrm{R}_{2}^{2}(8)$ dimer is by far the most stabilising pairwise interaction in any structure. In general, however, there is no straightforward correlation between intermolecular interaction energies of the pairwise motifs in the polymorphs and their frequency of occurrence in the multi-component set. The extensive SLFZ set provides a challenge for systematic geometrical comparison of crystal structures, and some observations are made on the methodology and consistency of the applied programs.

\section{Introduction}

Interest in the crystal structures and properties of molecular solids is, broadly speaking, driven by two main demands. On

\footnotetext{
${ }^{a}$ Yusuf Hamied Department of Chemistry, University of Cambridge, Lensfield Road, Cambridge, CB2 1EW,UK. E-mail:dh536@cam.ac.uk,adb29@cam.ac.uk

${ }^{b}$ School of Chemistry, Faculty of Engineering and Physical Sciences, University of Southampton, Southampton, SO17 1BJ, UK

$\dagger$ Electronic supplementary information (ESI) available: Set of standardised, energy-minimised structures in CIF format; list of the 96 crystal structures, including chemical identity, molecular diagrams and unit-cell parameters; refinement details for the 52 new crystal structures (CCDC 2120768-2120819); details of the DFT-D calculations; summaries of program output from CrystalCMP, COMPACK and XPAC; PIXEL energies for the polymorphs; pairwise motifs identified in the polymorphs and multi-component structures. For ESI and crystallographic data in CIF or other electronic format see DOI: 10.1039/d1ce01516h
}

the one hand are the manufacturing and patent aspects associated with the production and marketing of industrially important solid materials, exemplified particularly by pharmaceuticals. $^{1-9}$ On the other is the search for designer solids with utilisable properties, such as novel conjugated materials for use in organic solar cells, light-emitting diodes and transistors. ${ }^{10}$ Such studies relate to the broader topics of crystal engineering and crystal structure prediction (CSP). ${ }^{11-14}$ Research in these areas has developed to a point where crystal assembly can be designed to a significant extent, especially where intermolecular interactions are at the stronger end of the scale. ${ }^{15-17}$ Successes in CSP are also increasing at a steady rate and the current state of the art means that structures with many degrees of freedom and multi-component crystals can often be predicted, using a number of programs. ${ }^{13}$ Synergy between CSP and experimental studies has been 
realised in some cases ${ }^{18-20}$ and the long-term potential for application of machine learning and artificial intelligence to the design of solid forms is clear. ${ }^{21-24}$

In this context, one compound that we have studied extensively is sulfathiazole (SLFZ), a well-known active pharmaceutical ingredient (API; Scheme 1). ${ }^{25-27}$ Whilst in search of reliable crystallisation procedures for the SLFZ polymorphs, an extensive solvent-screening exercise was carried out, which yielded over 100 crystalline solvates. ${ }^{28}$ This represents one of the most extensive sets of multi-component crystals to be established for a single API, ${ }^{29}$ providing a rich opportunity to explore its solid-form landscape. This paper presents crystallographic data for the multi-component SLFZ crystal forms and discusses some initial efforts to analyse the structures. In total, the structure set comprises 91 multicomponent structures (52 new structures, 39 retrieved from the Cambridge Structural Database $(\mathrm{CSD})^{30}$ ) plus five SLFZ polymorphs. Systematic comparison of such a large set is a significant challenge which might be approached in various ways. The principal focus in this paper is on geometrical similarity, assessed using three generally available programs: COMPACK, ${ }^{31}$ (as implemented in Mercury $^{32}$ ), CrystalCMP ${ }^{33,34}$ and $X P a c .{ }^{35}$ Of particular interest is the comparison of results from the three different sources and the challenges that arise while seeking to establish consistent conclusions. The geometrical analysis is accompanied by calculation of intermolecular interaction energies in the polymorphs, with a view towards establishing the extent to which these might be correlated with transferability of pairwise motifs within the structure set. Seaton et al. have previously taken a similar approach with a more limited set of SLFZ salts. ${ }^{36}$ A complementary analysis of the SLFZ set based on hydrogenbond topology is planned for a subsequent paper.

\section{Experimental section}

\section{X-ray crystallography}

52 new crystal structures were obtained from single-crystal $\mathrm{X}$-ray diffraction measurements made on various instruments at the University of Southampton. Experimental and refinement details are provided in the ESI. $\uparrow$ The available data were in some cases of limited resolution and several

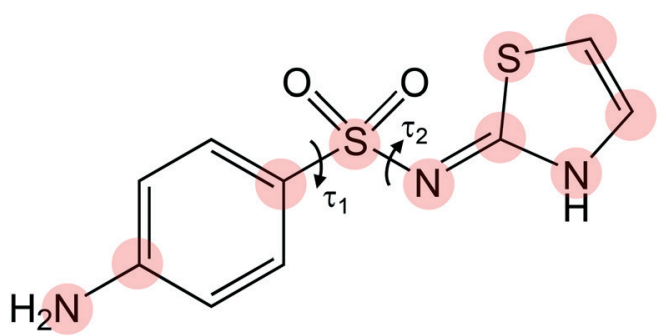

Scheme 1 Sulfathiazole (SLFZ), indicating rotatable torsions ( $\tau_{1}$ and $\tau_{2}$ ). Shaded atoms have unique (non-H) connectivity and are used to provide corresponding ordered sets for geometrical comparison. The imino tautomer shown is found exclusively in the structure set. The alternative amino tautomer is not seen. structures showed disorder of the solvent molecules. In all disordered cases, except $\mathbf{8}$, it was possible with suitable restraints to refine two distinct orientations of the solvent molecule, to give satisfactory coordinate sets for subsequent energy minimisation (see below). For $\mathbf{8}$, the location of the solvent molecule was clear, but the electron density appeared to comprise an overlay of several orientations which could not easily be resolved. The most prominent set of peaks in the electron density was modelled with restraints to provide a starting set for energy minimisation, but acceptable $R$-factors could only be produced in the X-ray refinement by application of the SQUEEZE algorithm. ${ }^{37}$ The structure of $\mathbf{8}$ is part of a large isostructural group (see Results section) and the solvent molecule is not involved in hydrogen bonding, so uncertainty in its exact position has no significant influence on the subsequent analysis.

\section{Numbering scheme and standardisation of the structures}

The literature contains conflicting numbering schemes for the SLFZ polymorphs. Table 1 shows the scheme applied here, ${ }^{38}$ with representative CSD refcodes. Throughout this paper, the suffix " $\mathbf{p}$ " is applied to indicate the polymorphs. The multi-component structures are labelled 1-91, with 1-59 being co-crystals, 60-88 being salts and 89-91 being other types (described in the Results section).

In each structure, a consistent atom numbering scheme is applied to SLFZ, as shown in Fig. 1. Since several structures have $Z^{\prime}>1$, a 2-digit code is adopted, where the first number identifies the molecule index and the second is the atom label within the molecule. The molecule has two torsion angles expected to show significant variation amongst the set, denoted $\tau_{1}$ and $\tau_{2}$ in Scheme 1. In the crystal, the molecule can exist in two pseudo-chiral conformations, leading to atropisomerism. ${ }^{39}$ For consistency in the standardised set, the SLFZ molecule in the asymmetric unit (or the molecule given index 1 in cases with $Z^{\prime}>1$ ) is chosen so that the thiazole ring lies to the left when the molecule is viewed along the bisector of the $\mathrm{SO}_{2}$ group with the $\mathrm{S}=\mathrm{O}$ bonds directed toward the viewer (Fig. 1), which corresponds to a negative value for $\tau_{2}$. This is referred to as the " $R$ " (reference) conformation, and the conformation of other

Table 1 Numbering scheme applied to the SLFZ polymorphs, with crystallographic information and representative CSD refcodes

\begin{tabular}{llllclll}
\hline & $\begin{array}{l}\text { CSD } \\
\text { refcode }\end{array}$ & $\begin{array}{l}\text { Space } \\
\text { group }\end{array}$ & \multicolumn{2}{l}{$\begin{array}{l}\text { Unit-cell } \\
\text { parameters }(\AA, \circ)\end{array}$} & & Vol $\left(\AA^{3}\right)$ & $Z / Z^{\prime}$ \\
\hline 1p & SUTHAZ16 & $P 2_{1} / c$ & 10.534 & 12.936 & 17.191 & 2230.8 & $8 / 2$ \\
& & & 90 & 107.77 & 90 & & \\
2p & SUTHAZ05 & $P 2_{1} / n$ & 10.399 & 15.132 & 14.280 & 2246.6 & $8 / 2$ \\
& & & 90 & 91.21 & 90 & & \\
3p & SUTHAZ17 & $P 2_{1} / c$ & 17.448 & 8.498 & 15.511 & 2120.0 & $8 / 2$ \\
& & & 90 & 112.81 & 90 & & \\
4p & SUTHAZ18 & $P 2_{1} / c$ & 8.193 & 8.538 & 15.437 & 1077.2 & $4 / 1$ \\
& & & 90 & 94.01 & 90 & & \\
5p & SUTHAZ19 & $P 2_{1} / n$ & 10.774 & 8.467 & 11.367 & 1036.5 & $4 / 1$ \\
& & & 90 & 91.65 & 90 & &
\end{tabular}




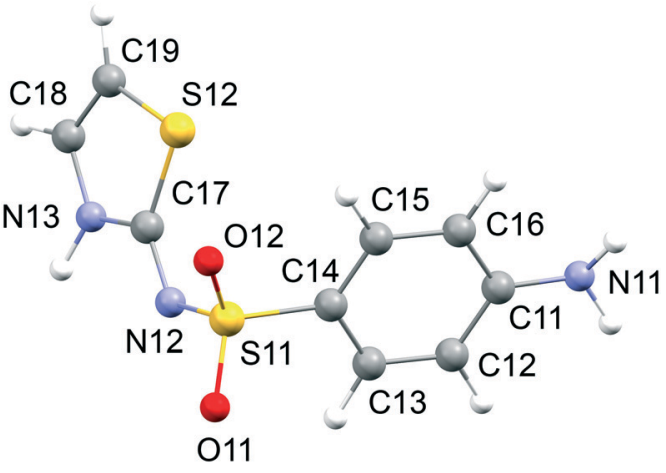

Fig. 1 Reference ("R") conformation of the SLFZ molecule applied in the standardised structures. The $\mathrm{S}=\mathrm{O}$ bond vectors are directed towards the viewer, with $\mathrm{O} 12$ uppermost and the thiazole ring to the left.

molecules is labelled $R$ or $S$ relative to that reference. The choice of the $R$ conformation is arbitrary; the purpose is to describe whether specific molecules have the same or different pseudo-chirality. In some of the noncentrosymmetric structures, experimental absolute structure determination indicated that the $S$ conformation is exclusively present in the crystal analysed, while others showed inversion twinning. All structures are converted to the $R$ conformation in the standardised set.

\section{Energy minimisation using DFT-D calculations}

Since the crystal structures originate from various sources and have a range of quality indicators, each structure was energy minimised using dispersion-corrected density functional theory (DFT-D) calculations. These optimisation methods have been established to reproduce correct crystal structures. ${ }^{40}$ For this work, they provide a "cleaned" data set in which all structures are placed on a common basis. Particular clarification is achieved in the positions of $\mathrm{H}$ atoms, which enables a more confident (automated) assessment of hydrogen bonding, to be discussed in a subsequent paper. Energy minimisation is also helpful where the X-ray structures contain poorly-resolved or disordered solvent molecules. All further discussion refers to the set of standardised and energy-minimised structures, which are provided in the ESI. $\dagger$ In some cases, it was necessary to reduce the space group symmetry of the X-ray structure to generate complete molecules, or to eliminate disorder of the solvent molecules. These cases are noted in the ESI.†

\section{Pairwise intermolecular interaction energies}

Pairwise intermolecular interaction energies were calculated for the polymorphs using the PIXEL methodology. ${ }^{41}$ Similar calculations have been published previously for $\mathbf{1 p}, \mathbf{3 p}, \mathbf{4 p}$ and $5 \mathbf{p}$ by Sovago et al. $^{42}$ The calculations were applied here to the DFT-D minimised structures, retaining the $\mathrm{H}$ atom positions. Tables of interaction energies, including symmetry notation consistent with the rest of the study, are provided in the ESI. $\dagger$ Conversion of the PIXEL output was implemented through a modified version of the processPIXEL utility. ${ }^{43}$

\section{Computer programs}

Geometrical comparison of the crystal structures was carried out using COMPACK ${ }^{31}$ (implemented as the Crystal Packing Similarity tool in Mercury $^{32}$ ), CrystalCMP ${ }^{33,34}$ and XPac. ${ }^{35}$ To permit comparison of the full solid-form landscape (i.e. polymorphs and multi-component forms), the comparisons are applied to the SLFZ molecules only. $\mathrm{H}$ atoms are excluded (enabling direct comparison of tautomers and different charge states) and partner molecules are omitted. On the basis of connectivity, 10 atoms are identified uniquely in each SLFZ molecule (Scheme 1), providing corresponding ordered sets of points for use in CrystalCMP and XPac. COMPACK does not require an ordered set of points to be defined.

\section{Symmetry notation}

To compare output between the various programs, the symmetry notation of $P_{L A T O N}{ }^{44}$ is adopted. Each SLFZ molecule in the asymmetric unit is designated by the symmetry code 1555_01 (and 1555_02, etc., where $Z^{\prime}>1$ ). The first digit identifies an applied symmetry operator by its position in a specified list, followed by encoding of any further translation along $x, y$ and $z$, respectively: 555 denotes no additional translation, 655 denotes +1 along $x, 545$ denotes -1 along $y$, etc. Where there is more than one molecule, an index is appended. For example, 3654_02 specifies the second molecule after application of the third symmetry operator in the input operator list, with a further translation of +1 along $x$ and -1 along $z$. The notation obviously depends on the defined sequence of symmetry operators, so care was taken to ensure that the sequences in the standardised CIFs are identical to those within PLATON. In general, the requirement for such consistency amongst inputs cannot form part of any robust automated methodology, but it is helpful here to achieve consistency between the various programs to be applied.

\section{Results and discussion}

\section{Range of the structure set}

The 91 multi-component structures comprise 59 co-crystals (containing neutral SLFZ and co-former molecules) and 29 salts (containing charged SLFZ and partner anions/cations) (see ESI $\dagger$ ). Three structures fall outside of this straightforward classification: 89 contains both neutral and charged SLFZ molecules, forming $\left[(\mathrm{SLFZ})_{2}\right]^{-}$units with sparteine cations; 90 contains $\mathrm{SLFZ}^{+}$with both anionic dinitrobenzoate and neutral dinitrobenzoic acid; 91 contains neutral SLFZ together with ion-separated adamantyl chloride. Amongst the salts, $\mathrm{SLFZ}^{-}$is roughly twice as common as $\mathrm{SLFZ}^{+}$, and all neutral SLFZ molecules are found as the imino tautomer. To enable a broad survey across the diverse structure set, the structural comparisons throughout this paper are applied to the 
standardised, DFT-D optimised structures, including only the non-H atoms of the SLFZ molecules.

A previous survey of the conformational characteristics of $N$-substituted arylsulfonamides ${ }^{45}$ identified two torsion angles expected to show significant variation, denoted $\tau_{1}$ and $\tau_{2}$ in Scheme 1. An analysis of these torsion angles for the SLFZ set (see ESI $\dagger$ ) shows that $\tau_{2}$ resembles a Gaussian distribution with mean $78^{\circ}$ and standard deviation $8^{\circ}$. Torsion angle $\tau_{1}$ also resembles a Gaussian with approximate mean $111^{\circ}$ and standard deviation $10^{\circ}$ but with a residual tail extending to higher values, populated principally by salts. A scatterplot of $\tau_{2}$ versus $\tau_{1}$ shows a loose cluster centred around the mean values of $\tau_{1}$ and $\tau_{2}$, with the extension to higher $\tau_{1}$ values seen clearly for the salts (ESI $\dagger$ ). The polymorphs fall mostly within the bulk cluster, except for molecule 1 of $2 \mathbf{p}\left(\tau_{1}=137.2, \tau_{2}=-98.6^{\circ}\right)$, which is an outlier due to its high $\tau_{2}$ value. On this basis, $2 \mathbf{p}$ might be distinguished as a conformational polymorph. ${ }^{46}$

\section{Overview of comparison methodology}

All of the applied comparison programs consider clusters of molecules built around a kernel molecule, thereby being independent of choices for the unit cell, space group, etc. Clusters of 15 molecules are typically considered to be sufficient to compare structures. The clusters being compared (A and B) are effectively aligned by overlaying the kernel molecules, then the remaining molecules in $\mathbf{A}$ and $\mathbf{B}$ are compared to each other using some geometrical criteria. An inversion-related copy of cluster $\mathbf{B}$ should also be tested, and separate clusters might be built for independent molecules in structures with $Z^{\prime}>1$. The result is a group of molecules in the two structures that are considered to match, with some quantitative measures of the geometrical similarity. The three applied programs differ in the details of their application and the nature of the results reported. Full details of the methodologies are given in the original papers, ${ }^{31,33-35}$ but the main differences are summarised as follows.

CrystalCMP produces a single continuous figure-of-merit, $\mathrm{PS}_{\mathrm{AB}}$, calculated from the distances and relative orientations of mapped molecules. ${ }^{33,34}$ A smaller value of $\mathrm{PS}_{\mathrm{AB}}$ indicates a greater degree of structural similarity. Clusters $\mathbf{A}$ and $\mathbf{B}$ are initially aligned to give the least-squares distance overlay of the atoms in the kernel molecules, then the remaining molecules in A and $\mathbf{B}$ are mapped by identifying the shortest distances between their centroids. A search fragment must be defined to compare molecules, and the $\mathrm{PS}_{\mathrm{AB}}$ value incorporates an RMSD measure between corresponding atoms. ${ }^{34}$ The final $\mathrm{PS}_{\mathrm{AB}}$ value is based on all (usually 15) mapped molecules.

COMPACK and XPac map molecules in a fundamentally different way, by considering local pairwise similarity. A shell is built around the kernel in the reference cluster A, comprising molecules "connected" by any intermolecular interatomic distance shorter than the sum of the van der Waals radii plus some tolerance. Each shell molecule in $\mathbf{A}$ is then compared to each molecule in cluster $\mathbf{B}$, and molecules are retained in the growing group of mapped molecules if an $\mathbf{A} \leftrightarrow \mathbf{B}$ match is established according to specified distance and angle criteria (which differ between the two programs). The process is continued to second-shell contacts for those molecules retained in the growing group until all molecules in cluster $\mathbf{A}$ have been visited. Full 3-D isostructurality is established in COMPACK if all (usually 15) molecules in cluster $\mathbf{A}$ are matched, and sub-structure similarity is indicated where only some molecules are matched. XPac interprets the established mappings to identify supramolecular constructs (SCs) within structure sets, comprising groups of matched molecules that may be $0-\mathrm{D}$ (isolated) or extend in 1-D, 2-D or 3-D. XPac also reports symmetry operators applied to generate each molecule within each SC, which can be helpful to identify them within a large set of structures and to compare with other programs such as PLATON or PIXEL. XPac, like CrystalCMP, requires a search fragment to be defined, while COMPACK establishes corresponding atoms automatically by comparing atom types and connectivity. In both COMPACK and XPAc, the requirement for threshold judgements during the mapping of molecules means that results depend on the chosen tolerances.

\section{Application of CrystalCMP}

The 10 unique non- $\mathrm{H}$ atoms identified in Scheme 1 were applied as the search fragment within CrystalCMP. Since this fragment omits atoms $\mathrm{C} 12 / \mathrm{C} 13 / \mathrm{C} 15 / \mathrm{C} 16$, the comparisons are not affected by the relative rotation of the phenyl ring (torsion angle $\tau_{1}$ ). The CrystalCMP dendrogram and accompanying similarity matrix for all 96 structures is included in the ESI. $\uparrow$ Groups of similar structures emerge, including one particularly large group, as shown in Fig. 2. In this group, 15 structures are clustered at $\mathrm{PS}_{\mathrm{AB}} \leq 5$ (links shaded dark or light green), and 5 further structures $(7,33,34,37,41)$ are linked to the group with larger $\mathrm{PS}_{\mathrm{AB}}$ values (links shaded orange). Structures 7 and 37 are quite closely similar to each other $\left(\mathrm{PS}_{\mathrm{AB}}=2.8882\right)$, but less closely related to the rest of the group. Inspection shows that most of these structures have directly comparable unit cells in space group $P 2_{1} / n$ (see ESI $\dagger$ ). For 41, the unit-cell volume is doubled on account of ordering of the solvent molecules (pyrrolidine-1-carbonitrile), but the SLFZ molecules alone are

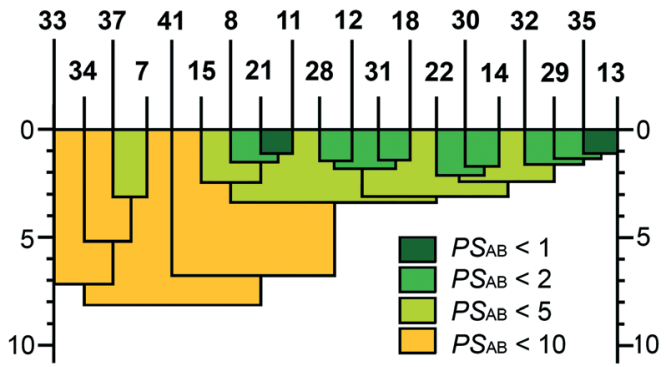

Fig. 2 Extract from the CrystalCMP dendrogram for the multicomponent SLFZ set. These structures (excluding 7 and 37) constitute a large 3-D isostructural group (group 1 in Table 2). Structures 7 and 37 form a separate group (group 2), as discussed in the text. 
described by the smaller unit cell in $P 2_{1} / n$, as for the rest of the group. Hence, 18 of the structures (excluding 7 and 37) constitute a 3-D isostructural group, denoted group 1 in Table 2. Although the isostructurality is clear on visual inspection (Fig. 3), there is considerable variation in the unitcell parameters amongst the group. The unit-cell volume (halved for 41) ranges from 1494 (28) to $1797 \AA^{3}$ (33) due to accommodating different solvent molecules. Thus, the SLFZ framework in this group shows considerable geometrical flexibility and CrystalCMP is effective to highlight the isostructurality despite these metric differences. All structures in group 1 are co-crystals, and the co-former molecules are not involved in conventional hydrogen-bonding with SLFZ (i.e. they are principally "space filling").

The structures of 7 and 37 are useful to illustrate the sensitivity and potential ambiguity of CrystalCMP. Visually, 7 and 37 appear similar as a pair, although the distortion of the unit cell is quite substantial (Fig. 4). The unit-cell parameters are comparable to group 1 , but 7 and 37 are described in space group $P 2_{1} / c$ rather than $P 2_{1} / n$ (for the same unit-cell setting). Comparing 7 or 37 to group 1, the structures look essentially identical when viewed along the $a$ axis (Fig. 5), and they contain consistent columns of hydrogen-bonded pairs running along $a$. However, neighbouring columns are shifted relative to each other along $a$. In group 1 , the relative position of neighbouring columns is established through $\mathrm{N} 11-\mathrm{H} \cdots \mathrm{O}$ hydrogen bonds between SLFZ molecules. In 7 and 37, these are replaced by hydrogen bonds to the solvent molecules ( $\gamma$-butyrolactone in 7 and pyridazine in 37), and the SLFZ molecules instead form O ${ }^{\cdots S} 12$ interactions. ${ }^{47,48}$ The geometrical difference between the molecular positions is subtle, but the difference in hydrogen bonding is clearly significant, and identifies 7 and 37 as a separate group (group 2). This conclusion is subsequently supported by results from COMPACK and XPAC (see below).

Visual inspection of the other groups in the CrystalCMP dendrogram identifies 3-D isostructurality as listed in Table 2. Isostructural groups exist for both co-crystals and salts, but there are no mixed groups. Some further observations can be made in relation to the methodology. In

Table 2 3-D isostructural groups identified amongst the multicomponent SLFZ structures. Consistent results are obtained using CrystalCMP, COMPACK and XPaC

\begin{tabular}{lll}
\hline Group 1 & $\{\mathbf{8}, \mathbf{1 1}, \mathbf{1 2}, \mathbf{1 3}, \mathbf{1 4}, \mathbf{1 5}, \mathbf{1 8}, \mathbf{2 1}, \mathbf{2 2}$, & Co-crystals \\
& $\mathbf{2 8}, \mathbf{2 9}, \mathbf{3 0}, \mathbf{3 1}, \mathbf{3 2}, \mathbf{3 3}, \mathbf{3 4}, \mathbf{3 5}, \mathbf{4 1}\}$ & \\
Group 2 & $\{\mathbf{7}, \mathbf{3 7}\}$ & Co-crystals \\
Group 3 & $\{\mathbf{5 6}, \mathbf{5 7}, \mathbf{5 8}, \mathbf{5 9}\}$ & Co-crystals \\
Group 4 & $\{\mathbf{6 2}, \mathbf{6 3}, \mathbf{7 2}\}$ & Salts \\
Group 5 & $\{\mathbf{1 6}, \mathbf{1 7}, \mathbf{1 9}\}$ & Co-crystals \\
Group 6 & $\{\mathbf{7 1}, \mathbf{8 5}\}$ & Salts \\
Group 7 & $\{\mathbf{6 9}, \mathbf{7 3}\}$ & Salts \\
Group 8 & $\{\mathbf{2 5}, \mathbf{4 7}\}$ & Co-crystals \\
Group 9 & $\{3 \mathbf{3 8}, \mathbf{5 2}\}$ & Co-crystals \\
Group 10 & $\{39, \mathbf{4 0}\}$ & Co-crystals \\
Group 11 & $\{\mathbf{7 7}, \mathbf{7 9}\}$ & Salts \\
Group 12 & $\{\mathbf{6 6}, \mathbf{8 8}\}$ & Salts
\end{tabular}

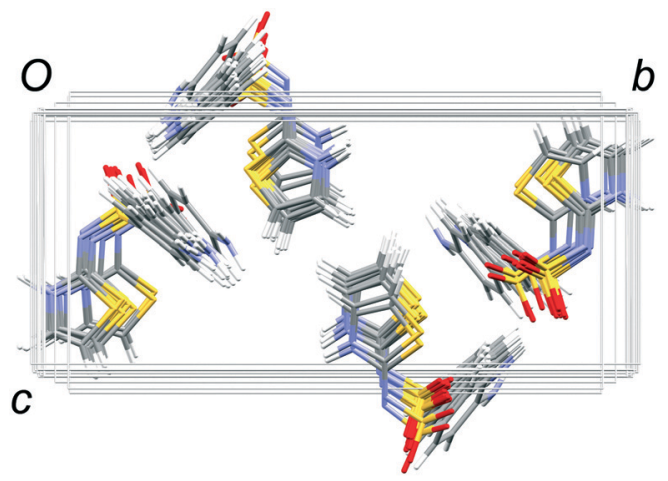

Fig. 3 Overlay of the SLFZ molecules in group 1, viewed down the a axis. 17 structures are shown (41, having a doubled unit cell, is omitted for clarity). The metric variability in the structures is apparent.
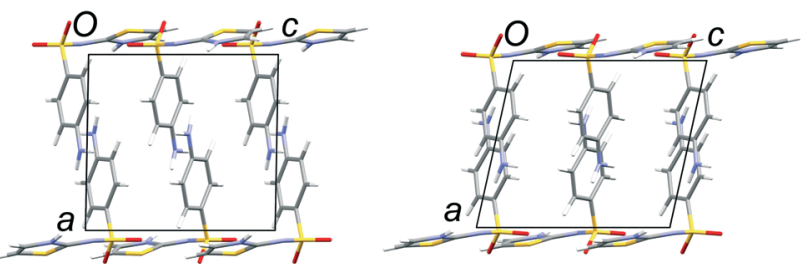

Fig. 4 Arrangement of SLFZ molecules in 7 (left) and 37 (right). The structures contain identical 2-D sections in the bc planes, although adjacent layers are offset and the unit cell is sheared due to accommodating different solvent molecules $(\gamma$-butyrolactone in 7 and pyridazine in 37 ).

the dendrogram, 38, 52 and $\mathbf{5 0}$ are linked at $\mathrm{PS}_{\mathrm{AB}} \approx 12$. These structures resemble group 1/group 2 in that $\mathbf{3 8}$ and $\mathbf{5 2}$ are isostructural, but $\mathbf{5 0}$ is subtly different. As for group 1/group 2, identical 1-D columns exist in all three structures along the $a$ axis, but neighbouring columns in $\mathbf{5 0}$ are shifted by $\frac{1}{2} a$ compared to the other two. Again, this is driven by the occurrence of $\mathrm{N} 11-\mathrm{H} \cdots \mathrm{O}$ hydrogen bonds between SLFZ molecules in 50, which are replaced by $\mathrm{N}-\mathrm{H} \cdots$ solvent hydrogen bonds in $\mathbf{3 8}$ and $\mathbf{5 2}$. Hence, $\mathbf{5 0}$ is not included in group 9. For group 12, comprising 66 and 88, the structures are clearly isostructural on visual inspection, but their similarity measure $\left(\mathrm{PS}_{\mathrm{AB}}=14.9780\right)$ is significantly larger than some of the cases deemed not to be isostructural. Given the clear visual similarity between 66 and 88, the value of $\mathrm{PS}_{\mathrm{AB}}$ is surprisingly high, and could indicate that corresponding molecules may not be appropriately mapped. In general, the geometrical $\mathrm{PS}_{\mathrm{AB}}$ measure is clearly helpful to identify cases of potential 3-D isostructurality, but it is difficult to select a consistent cut-off value for fully automated grouping of the SLFZ set.

\section{Application of COMPACK}

Using COMPACK, it was found that distance/angle tolerances of $30 \% / 30^{\circ}$ (extended from the default $20 \% / 20^{\circ}$ ) were required to identify the established cases of 3-D isostructurality in Table 2. Applying a full 96 vs. 96 

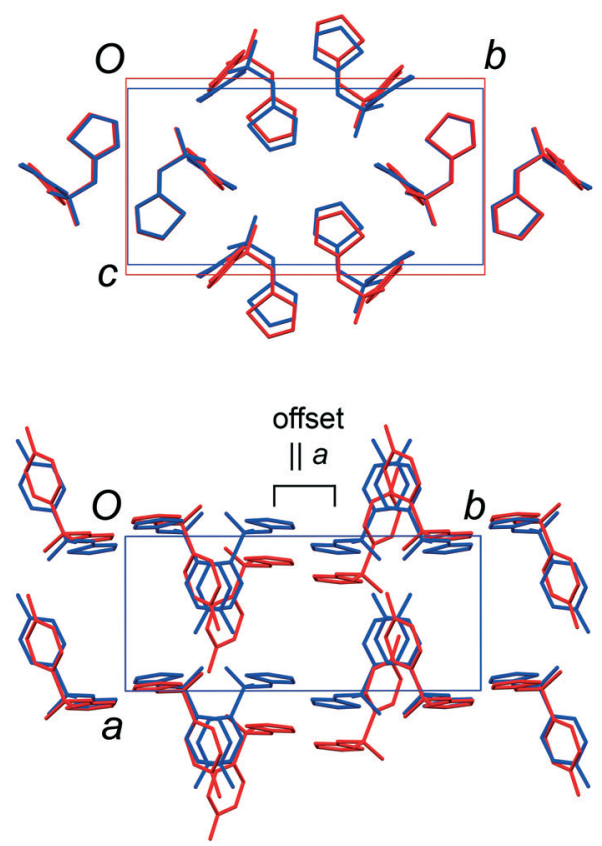

Fig. 5 SLFZ molecules in 37 (blue) compared to group 1 (red; 33 is shown as a representative example). The structures look essentially identical in projection along the a axis (top), but adjacent columns of $\mathrm{H}$-bonded pairs are offset in $\mathbf{3 3}$ compared to $\mathbf{3 7}$ (bottom) to produce a different overall hydrogen-bonded network. Pyridazine solvent molecules, omitted from these diagrams, accept hydrogen bonds from SLFZ in 37.

comparison with these tolerances followed by automatic grouping reproduces Table 2 exactly, including separation of groups 1 and 2 (see $\operatorname{ESI} \dagger$ ). Hence, COMPACK is marginally more convenient than CrystalCMP for identifying 3-D isostructural groups consistent with visual expectations. Within group 1, however, not all pairwise matches are made at the 15-molecule level. In particular, 33 and 34 fail to match fully with several other structures in the group, indicating that their similarity with the group is close to the upper threshold for acceptance. This is consistent with the relatively larger unit-cell volume of $\mathbf{3 3}$ and $\mathbf{3 4}$ (see ESI†) and also with the fact that both are linked to group 1 at a higher $\mathrm{PS}_{\mathrm{AB}}$ value in the CrystalCMP dendrogram (Fig. 2). To achieve a complete 15-molecule match between all pairs of structures in group 1 , it is necessary to increase the COMPACK tolerances to $45 \%$ / $40^{\circ}$. However, such a liberal tolerance fails to distinguish between groups 1 and 2, so it is again difficult to identify one set of COMPACK tolerances that would produce all pairwise matches consistent with Table 2.

Although COMPACK automatically groups structures having 15-molecule similarity, it is a substantial manual task to distil the information for sub-structure similarity. An example has been published for 50 structures containing carbamazepine. ${ }^{49}$ For the SLFZ set, 2234 out of 4560 pairwise comparisons identify some match beyond the kernel molecule, so a fully comprehensive description of the COMPACK output is impractical. The discussion below is restricted to a few illustrative examples.
Considering pairwise matches down to the 9-molecule level yields only a few new links between structures in addition to the groups identified in Table 2. An interesting methodological feature emerges, however. Structure 48 shows 13-molecule similarity with group 3, while 64 matches the same group at the apparently less similar 8-molecule level. Visual inspection shows that both matches actually correspond to the same structural feature, which is a 2-D hydrogen-bonded layer (Fig. 6). For 64 versus 56, one clearly corresponding molecule within the layer just fails to match at the $30 \% / 30^{\circ}$ tolerance level (so the match essentially involves 9 molecules), but the remaining difference in the number of matched molecules is not due to tolerances. Rather, it is a consequence of the relative positions of the common SLFZ layers. In 48, they are well separated due to inclusion of 18-crown-6 and acetonitrile in the multicomponent structure. As a result, 13 of the 15 SLFZ molecules in the initial cluster built for $\mathbf{4 8}$ belong to the common 2-D layer, and the different relative positions of the layers compared to group 3 is revealed by only 2 mismatched molecules (Fig. 7, top). For 64, the common SLFZ layers are in direct contact, and only 8 molecules in the initial cluster around the kernel molecule belong to the common 2-D layer. Now the difference between layers is revealed by 6 mismatched molecules in neighbouring layers (Fig. 7, bottom). This example highlights that it is not straightforward to interpret the substructure information generated by COMPACK, or even to state immediately that a greater number of matched molecules corresponds to a higher degree of structural similarity. Although it is possible to vary the size of the initial cluster in COMPACK, this type of discrepancy will remain in situations where common substructure motifs are arranged in significantly different ways. Multi-component structures will be more susceptible to such effects because the target molecules are likely to be dispersed more widely to accommodate the partner molecules.

Amongst the other structures matched at the 9-molecule level or greater in COMPACK, a polytypic relationship is identified between polymorphs $\mathbf{3 p}, \mathbf{4 p}$ and $\mathbf{5 p}$ (Table 1), whereby consistent 2-D layers lie in the (100) planes for $3 \mathbf{p}$

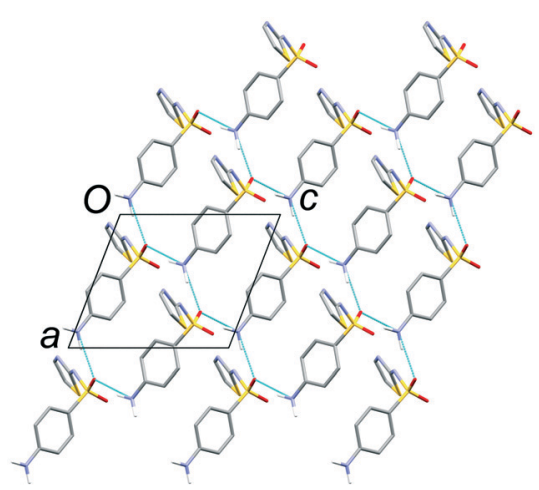

Fig. 6 Projection onto the plane of the common 2-D layer of SLFZ molecules identified in the structures of group 3, 48 and 64 (structure 48 is shown). The dashed lines indicate $\mathrm{N}-\mathrm{H} \cdots \mathrm{O}$ hydrogen bonds. 

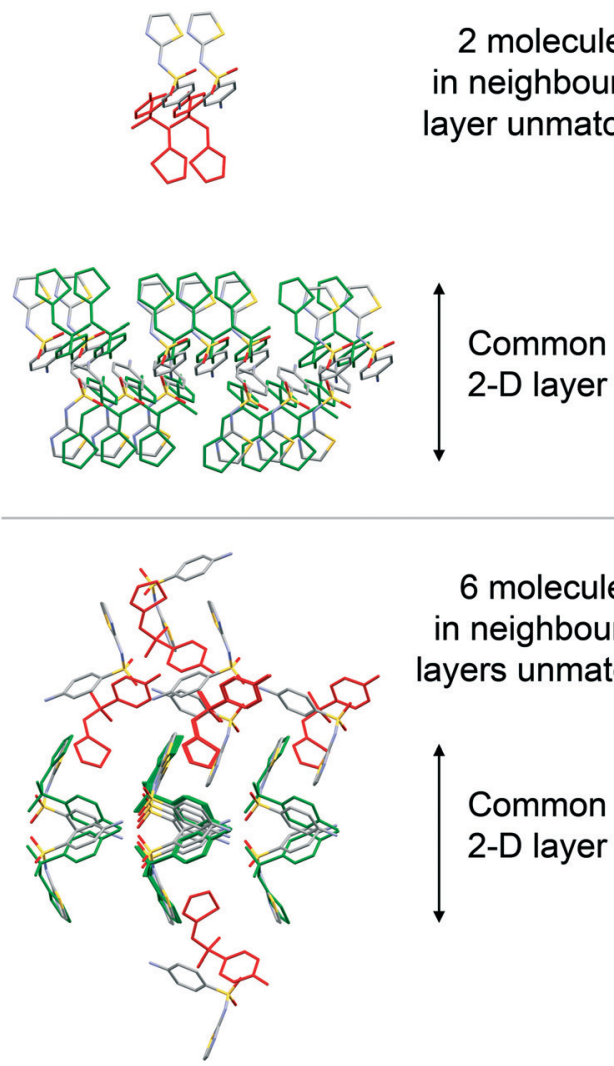

\section{6 molecules in neighbouring layers unmatched}

\section{Common} 2-D layer
Fig. 7 15-Molecule clusters in 48 (top) and 64 (bottom), both compared to 56. Matching molecules are coloured green and unmatched molecules are red. Solvent molecules ((18-crown-6)/ acetonitrile in 48 and 2-methyl-2-imidazoline in 64) are omitted.

and $4 \mathbf{p}$ and in the (10-1) planes for $\mathbf{5 p}$ (Fig. 8). For $\mathbf{4 p}$, the layers are stacked by translation along the $a$ axis (AAAA stacking pattern), while in $\mathbf{5 p}$, every second layer is mirrored perpendicular to the $b$ axis ( $A B A B$ stacking pattern). Polymorph $3 \mathbf{p}$ shows an intermediate $A A B B$ pattern. The various pairwise matches between $3 \mathbf{p} / \mathbf{4} \mathbf{p} / \mathbf{5} \mathbf{p}$ in COMPACK range between 9 and 13 matched molecules. This is clearly helpful to draw attention to the similarity between the structures, but manual inspection is still required to extract details of the polytypism.

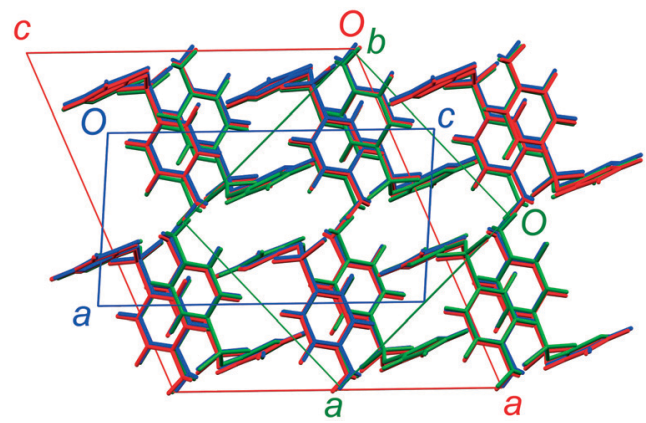

Fig. 8 Polytypic relationship between the structures of $3 p$ (red), 4p (blue) and $5 p$ (green). The structures share common 2-D layers (horizontal), but have different stacking sequences.

\section{Application of XPac}

A full 96 vs. 96 comparison was carried out using XPac, with "high" tolerances $\left(\delta_{\mathrm{ang}}=12, \delta_{\mathrm{dhd}}=18^{\circ}\right)$. The 3-D SCs identified within the set are consistent with the 3-D isostructural groups listed in Table 2. Most pairwise comparisons within each group yielded a 3-D SC, except for $28 v s$. 33 in group 1, which appears just to exceed the tolerance limits and returns 2-D similarity. Groups 1 and 2 are distinguished at the applied tolerance level, returning a common 2-D SC in the ac planes (a single layer of hydrogen-bonded pairs, running vertically in Fig. 5). Comparison of the isostructural $\mathbf{3 9}$ and $\mathbf{4 0}$ also returns a 2-D $\mathrm{SC}$, but this is not due to tolerances. Rather, it is a reflection of the cluster building process, similar to that described for COMPACK. Structures 39 and $\mathbf{4 0}$ show a long $c$ axis $(\sim 39 \AA)$, which means that the generated clusters do not contain any molecules related by translation along $c$. The problem can be eliminated by increasing the initial cluster size, but again it raises a methodological question of how an initial cluster of molecules might best be defined without manual intervention.

Across the whole structure set, common 2-D SCs are generally restricted to groups of only two or three structures and there are no 2-D SCs that are observed especially frequently. One example links groups 8 and 10, which contain a common 2-D SC comprising SLFZ molecules linked by $\mathrm{N}-\mathrm{H} \cdots \mathrm{O}$ hydrogen bonds into polar layers (Fig. 9). In 25/ 47, the SLFZ molecules in neighbouring layers are linked by $\mathrm{N}-\mathrm{H} \cdots \mathrm{N}$ hydrogen bonds forming an inversion-symmetric $\mathrm{R}_{2}^{2}(8)$ motif (discussed further below). The structure adopted by $39 / 40$ is more complex, showing alternating polar and non-polar layers (Fig. 9). An $\mathrm{R}_{2}^{2}(8)$ motif is again found between neighbouring layers, but with $C_{2}$ symmetry rather than inversion symmetry.

Transferable 1-D SCs are more common within the set. For example, the arrangement along the $a$ axis of $4 \mathbf{p}$ is built from $\mathrm{N}-\mathrm{H} \cdots \mathrm{O}$ hydrogen bonds between SLFZ molecules related by translation (Fig. 10). XPac identifies this 1-D SC in 14 co-crystals, 7 salts and one other structure, totalling $c a$.

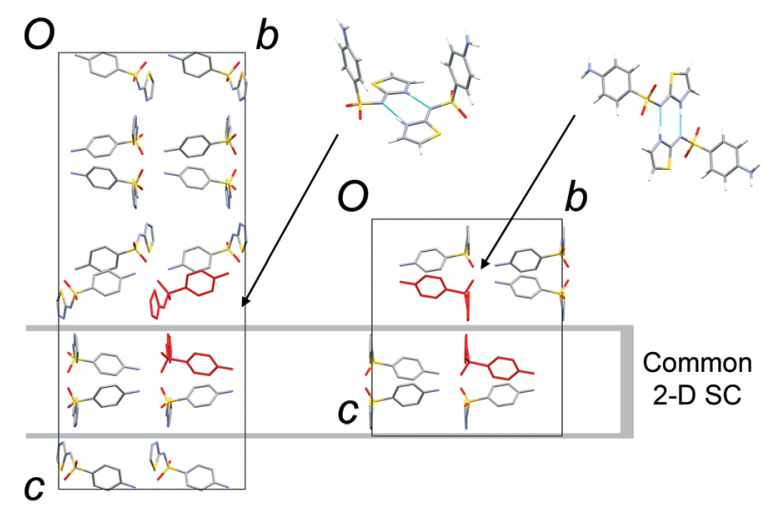

Fig. 9 Common 2-D supramolecular construct in the structures of group 10 (left; 39 shown) and group 8 (right; 47 shown). The molecules linked by $\mathrm{N}-\mathrm{H} \cdots \mathrm{N} \mathrm{R} 2(8)$ motifs discussed in the text are highlighted. Solvent molecules (1,4-dioxane in 47 and propionitrile in 39) are omitted. 
(a)

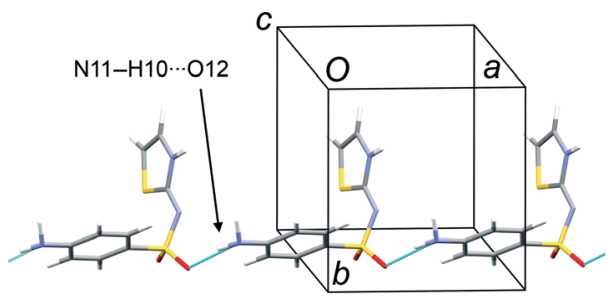

(b)

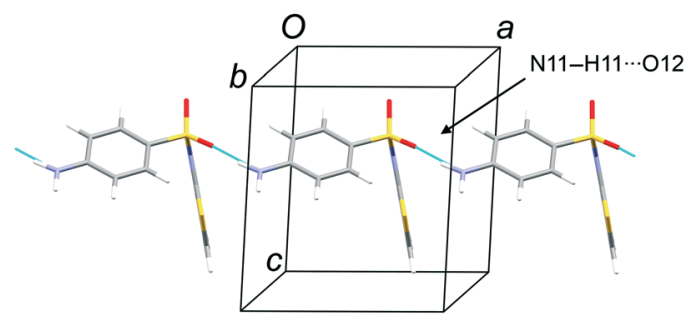

Fig. 10 1-D SC built from $\mathrm{N}-\mathrm{H} \cdots \mathrm{O}$ hydrogen bonds between SLFZ molecules related by translation: (a) along the a axis in $4 p$; (b) along the $a$ axis in group 1 ( 8 is shown).

one quarter of the multi-component crystals. An identical arrangement of hydrogen-bonded aminobenzene rings is seen along the $a$ axis of the group 1 structures, plus two other co-crystals $(20,37)$ and two salts $(\mathbf{7 0}, \mathbf{8 4})$, again totalling $c a$. one quarter of the multi-component set. Hence, in total, almost one half of the multi-component crystals adopt this hydrogen-bonding arrangement. The two 1-D SCs are geometrically different because the $\mathrm{N}-\mathrm{H} \cdots \mathrm{O}$ hydrogen bonds are formed either by H10 (in 4p; Fig. 10(a)) or H11 (in group 1; Fig. 10(b)), so that the direction of the translation relative to the SLFZ molecule is different. A closer look at some of the structures reveals the possibility for a subtle change in hydrogen bonding within these 1-D SCs. For example, the N$\mathrm{H} 10$ bond in 2 points clearly at N12 rather than O11 (Fig. 11). In some of the salts $(75,83,86)$, the amino group is protonated, and the $\mathrm{NH}_{3}{ }^{+}$group clearly interacts with both O11 and N12. It is perhaps to be expected that these transferable SCs should be built from hydrogen bonds, but the structure set also contains other 1-D SCs that are not based on hydrogen bonding, e.g. see Fig. 12 .

To summarise the extensive XPac output, a Hasse diagram might typically be constructed, showing the relationships between SCs identified in all structures. ${ }^{35,50,51}$ A complete diagram for the SLFZ set would be extraordinarily complex,

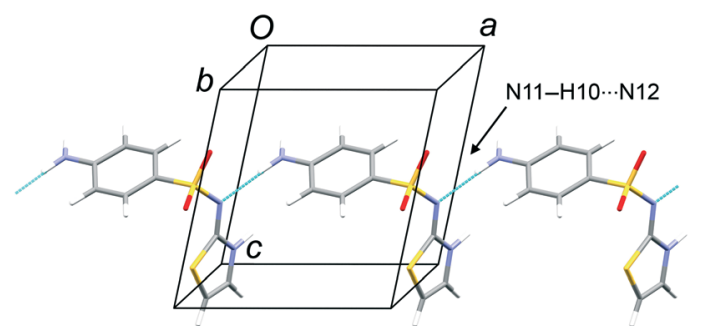

Fig. 11 1-D SC in 2, forming $\mathrm{N}-\mathrm{H} \cdots \mathrm{N}$ hydrogen bonds between translated SLFZ molecules.

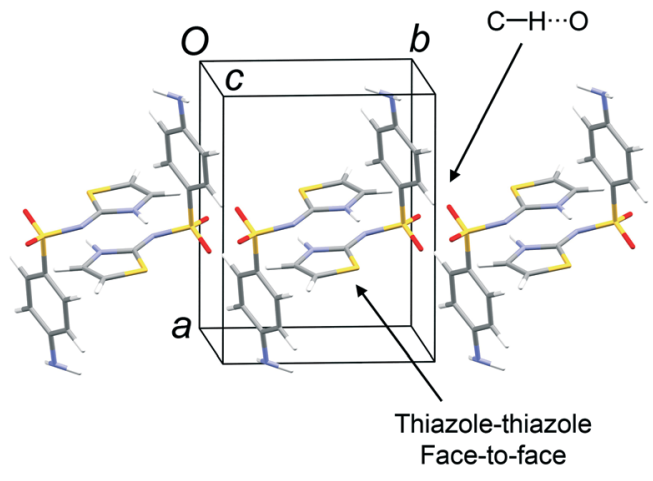

Fig. 12 Thiazole-thiazole and $\mathrm{C}-\mathrm{H} \cdots \mathrm{O}$ dimer interactions produce a 1-D supramolecular construct common to the structures of $2 p, 3 p, 4 p$ and $5 p$ ( $5 p$ is shown).

however, and the largely manual task of constructing it is forbidding. Details of the XPac comparison between the polymorphs and multi-component structures (5 vs. 91) are included in the ESI. Further description of the XPac output is deferred for a potential additional study.

\section{Pairwise intermolecular interactions in the polymorphs and multi-component structures}

A "bottom up" approach to structural similarity, which is effectively implemented in the molecule mapping processes of COMPACK and XPac, involves local matching of molecular pairs. From a chemical perspective, such an analysis of SLFZ across the structure set should provide insight into the balance between SLFZ-SLFZ and SLFZ-solvent interactions. The information is output directly by XPac, which identifies molecular pairs on the basis of their symmetry labels within identified SCs. Alternatively, the "Crystal Packing Feature" search within Mercury can be applied to the set, using a given molecular pair extracted from one of the structures. A combination of these two methods identified 15 pairwise SLFZ-SLFZ interactions that occur in the polymorphs and at least one multi-component structure (Table 3). The tolerancebased approach inevitably produces some inconsistency between the results obtained using XPac and Mercury, but Table 3 provides a fair guide to the relative frequencies of occurrence. The geometrical analysis is augmented by PIXEL intermolecular interaction energies calculated for each pairwise motif (see ESI $\dagger$ ).

The pairwise motif seen most frequently is the centrosymmetric $\mathrm{R}_{2}^{2}(8)$ dimer formed by a complementary pair of $\mathrm{N}-\mathrm{H} \cdots \mathrm{N}$ hydrogen bonds. This was also highlighted in the study by Seaton et al. ${ }^{36}$ The PIXEL calculations confirm that this is by far the most stabilising pairwise interaction in any of the polymorphs, and it occurs in roughly half of the multicomponent structures, including the large isostructural group 1. An alternative $C_{2}$-symmetric motif with the same $\mathrm{R}_{2}^{2}(8)$ hydrogen-bonding pattern has been mentioned earlier (Fig. 9). Since the $\mathrm{R}_{2}^{2}(8)$ motif requires $\mathrm{N} 13$ to be protonated, it is seen only in the co-crystals, and in total $c a$. $80 \%$ of the co-crystals 
Table 3 Frequently occurring pairwise interactions identified within the SLFZ polymorphs and at least one multi-component structure. Results are derived from $X P a c$ and Mercury. The quoted range of intermolecular interaction energies refers to different instances of the same pairwise motif within the polymorphs. A full list, with diagrams, is given in the ESI

\begin{tabular}{lllll}
\hline Motif & $\begin{array}{l}\text { Found in } \\
\text { polymorph }\end{array}$ & $\begin{array}{l}\text { No. of } \\
\text { structures }\end{array}$ & H-Bond? & $\begin{array}{l}\text { PIXEL interaction } \\
\text { energy }\left(\mathrm{kJ} \mathrm{mol}^{-1}\right)\end{array}$ \\
\hline $\mathbf{A}$ & $\mathbf{1 p}$ & 42 & $\mathrm{Y}$ & -147.3 to -136.7 \\
$\mathbf{B}$ & $\mathbf{3 p}, \mathbf{4 p}$ & 25 & $\mathrm{Y}$ & -33.2 to -30.1 \\
$\mathbf{C}$ & $\mathbf{2 p ,} \mathbf{3 p}, \mathbf{4 p}$ & 17 & $\mathrm{~N}$ & -50.4 to -35.9 \\
$\mathbf{D}$ & $\mathbf{2 p}$ & 15 & $\mathrm{Y}$ & -39.6 to -39.5 \\
$\mathbf{E}$ & $\mathbf{3 p}, \mathbf{4 p}, \mathbf{5 p}$ & 11 & $\mathrm{~N}$ & -31.9 to -30.1 \\
$\mathbf{F}$ & $\mathbf{3 p}, \mathbf{5 p}$ & 11 & $\mathrm{~N}$ & +19.5 \\
$\mathbf{G}$ & $\mathbf{1 p}$ & 9 & $\mathrm{~N}$ & +15.7 \\
$\mathbf{H}$ & $\mathbf{3 p}, \mathbf{5 p}$ & 8 & $\mathrm{~N}$ & -22.4 to -22.2 \\
$\mathbf{I}$ & $\mathbf{2 p}, \mathbf{3 p}, \mathbf{4 p , 5 p}$ & 8 & $\mathrm{~N}$ & -48.7 to -36.7 \\
\hline
\end{tabular}

contain either the centrosymmetric or $C_{2}$-symmetric $\mathrm{R}_{2}^{2}(8)$ motif. In the 12 structures where the $\mathrm{R}_{2}^{2}(8)$ motif is not seen, all but one make an $\mathrm{N}-\mathrm{H} \cdots \mathrm{N} / \mathrm{O}$ hydrogen bond to the solvent molecule. The sole exception is 10, where N13 makes an N$\mathrm{H} \cdots \mathrm{O}$ interaction to another SLFZ molecule. Hence, the cocrystals are dominated by the $\mathrm{R}_{2}^{2}(8)$ motif, but the probability of its formation is reduced where the co-former molecule is able to accept an $\mathrm{N}-\mathrm{H} \cdots \mathrm{N} / \mathrm{O}$ hydrogen bond.

In most cases, the pairwise interaction energies (assessed only for the polymorphs) are consistent for a given pair found in different structures, but some instances were identified where a subtle change in geometry has quite a significant effect on the resulting interaction energy. For example, the structures of $\mathbf{2 p}, \mathbf{3 p}, \mathbf{4 p}$ and $\mathbf{5 p}$ contain a common 1-D motif along one lattice direction, comprising two alternating SLFZSLFZ pairwise interactions (Fig. 12): (i) a centrosymmetric "closed" dimer involving face-to-face contact between thiazole rings, and (ii) a centrosymmetric pair involving $\mathrm{C}-\mathrm{H} \cdots \mathrm{O}$ interactions between aminobenzene rings. The geometries and interaction energies are consistent in $\mathbf{3 p}, \mathbf{4 p}$ and $\mathbf{5 p}$, but 2p shows a subtle geometrical distortion that affects both interactions. For the thiazole-thiazole pair, a greater degree of face-to-face overlap in $\mathbf{2 p}$ gives a larger repulsion term in the PIXEL energy and changes the total interaction energy from $c a$. -46 to $-38 \mathrm{~kJ} \mathrm{~mol}^{-1}$. For the $\mathrm{C}-\mathrm{H} \cdots \mathrm{O}$ interaction, the change in geometry in $\mathbf{2 p}$ is visually more subtle, but the centroid-centroid distance decreases by $c a .0 .2 \AA$ and the total interaction energy changes from $c a$. -48 to $-37 \mathrm{~kJ} \mathrm{~mol}^{-1}$. This example illustrates that the premise of transferable pairwise motifs, each with a well-defined interaction energy, must be viewed flexibly.

\section{Conclusions}

The new crystallographic data presented in this paper, combined with existing structures in the CSD, establishes a set of 96 crystal structures containing the active pharmaceutical ingredient (API) sulfathiazole (SLFZ). This is one of the largest groups of crystal structures currently available for any API, providing an unusually broad view of its solid-form landscape.
Identifying and describing structural similarity in this extensive set is challenging. This paper has focussed on available programs to assess geometrical similarity: CrystalCMP, COMPACK and XPac. Each program provides valuable results, but they depend on the applied metric measures/tolerances, and it remains difficult and time-consuming to synthesise the output to yield consistent and coherent conclusions, particularly regarding sub-structure similarity. Some aspects of the methodology also seem specifically less suitable for multicomponent structures.

For the SLFZ set, some confident conclusions can be drawn. For example, 3-D isostructural groups amongst the multi-component structures are robustly established. Some transferable supramolecular constructs have also been shown, although a comprehensive overview for the whole structure set is still to be addressed. Common pairwise motifs are identified in the polymorphs and multicomponent structures, some of which are based on conventional hydrogen bonding, and some of which are not. Although PIXEL calculations confirm that frequently occurring pairwise motifs are generally quite strongly stabilising, some less stabilising and even destabilising pairs are transferred, and there are numerous more stabilising interactions in the polymorphs that are not seen in the multi-component structures. Hence, there is no straightforward correlation between interaction energy and transferability of a given pairwise motif between the polymorphs and multi-component structures.

There is undoubtedly a great deal more knowledge to be extracted from the SLFZ structure set. A planned subsequent paper will augment this geometrical study with a complementary topological analysis of hydrogen bonding. Many more questions might be considered. For example, can the structure set reveal why SLFZ should be so prolific in forming multi-component crystal forms? Is it a quantifiable function of its shape and/or propensity to form H-bonded networks, or is it simply proportional to the time that has been spent looking? What can be learned about the likelihood of SLFZ forming a multi-component crystal with a given solvent/partner molecule? Are 91 known multicomponent structures sufficient to make meaningful conclusions, or do we need more? These types of questions are directly relevant to the practical task of "de-risking" the solid-form selection process, for example in pharmaceutical production. It is hoped that the SLFZ set will be valuable in this and other similar contexts.

\section{Conflicts of interest}

There are no conflicts to declare.

\section{Acknowledgements}

We are grateful to Dr Thomas Gelbrich, University of Innsbruck, for providing the XPac program, and we acknowledge his key contributions to the comparison 
methods that we have applied. We also thank Dr Graham Tizzard, University of Southampton, for helpful discussions.

\section{Notes and references}

1 Computational Pharmaceutical Solid State Chemistry, ed. Y. A. Abramov, Wiley, Hoboken, New Jersey, 2016.

2 J. Bernstein, Polymorphism in Molecular Crystals, Oxford University Press, Oxford, 2nd edn, 2020.

3 A. D. Bond, Pharmaceutical Crystallography: A Guide to Structure and Analysis, Royal Society of Chemistry, Cambridge, 2019.

4 H. G. Brittain, J. Pharm. Sci., 2012, 101, 464-484.

5 S. R. Byrn, R. R. Pfeiffer and J. G. Stowell, Solid-State Chemistry of Drugs, SSCI, Inc., West Lafayette, Indiana, USA, 2nd edn, 1999.

6 S. Datta and D. J. W. Grant, Nat. Rev. Drug Discovery, 2004, 3, 42-57.

7 Polymorphism: in the Pharmaceutical Industry, ed. R. Hilfiker, Wiley-VCH, Weinheim, 2006.

8 Handbook of Industrial Crystallization, ed. A. S. Myerson, D. Erdemir and A. Y. Lee, Cambridge University Press, Cambridge, 2019.

9 Handbook of Pharmaceutical Salts: Properties Selection and Use, ed. P. H. Stahl and C. G. Wermuth, Wiley-VCH, Weinheim, 2002.

10 H. Bronstein, C. B. Nielsen, B. C. Schroeder and I. McCulloch, Nat. Rev. Chem., 2020, 4, 66-77.

11 C. Bissantz, B. Kuhn and M. Stahl, J. Med. Chem., 2010, 53, 5061-5084.

12 A. K. Nangia and G. R. Desiraju, Angew. Chem., Int. Ed., 2019, 58, 4100-4107.

13 A. M. Reilly, R. I. Cooper, C. S. Adjiman, S. Bhattacharya, A. D. Boese, J. G. Brandenburg, P. J. Bygrave, R. Bylsma, J. E. Campbell, R. Car, D. H. Case, R. Chadha, J. C. Cole, K. Cosburn, H. M. Cuppen, F. Curtis, G. M. Day, R. A. DiStasio, A. Dzyabchenko, B. P. van Eijck, D. M. Elking, J. A. van den Ende, J. C. Facelli, M. B. Ferraro, L. Fusti-Molnar, C. A. Gatsiou, T. S. Gee, R. de Gelder, L. M. Ghiringhelli, H. Goto, S. Grimme, R. Guo, D. W. M. Hofmann, J. Hoja, R. K. Hylton, L. Iuzzolino, W. Jankiewicz, D. T. de Jong, J. Kendrick, N. J. J. de Klerk, H. Y. Ko, L. N. Kuleshova, X. Y. Li, S. Lohani, F. J. J. Leusen, A. M. Lund, J. Lv, Y. M. Ma, N. Marom, A. E. Masunov, P. McCabe, D. P. McMahon, H. Meekes, M. P. Metz, A. J. Misquitta, S. Mohamed, B. Monserrat, R. J. Needs, M. A. Neumann, J. Nyman, S. Obata, H. Oberhofer, A. R. Oganov, A. M. Orendt, G. I. Pagola, C. C. Pantelides, C. J. Pickard, R. Podeszwa, L. S. Price, S. L. Price, A. Pulido, M. G. Read, K. Reuter, E. Schneider, C. Schober, G. P. Shields, P. Singh, I. J. Sugden, K. Szalewicz, C. R. Taylor, A. Tkatchenko, M. E. Tuckerman, F. Vacarro, M. Vasileiadis, A. VazquezMayagoitia, L. Vogt, Y. C. Wang, R. E. Watson, G. A. de Wijs, J. Yang, Q. Zhu and C. R. Groom, Acta Crystallogr., Sect. B: Struct. Sci., Cryst. Eng. Mater., 2016, 72, 439-459.

14 P. A. Wood, T. S. G. Olsson, J. C. Cole, S. J. Cottrell, N. Feeder, P. T. A. Galek, C. R. Groom and E. Pidcock, CrystEngComm, 2013, 15, 65-72.
15 R. N. Costa, D. Choquesillo-Lazarte, S. L. Cuffini, E. Pidcock and L. Infantes, CrystEngComm, 2020, 22, 7460-7474.

16 D. Loveland, B. Kailkhura, P. Karande, A. M. Hiszpanski and T. Y. J. Han, J. Chem. Inf. Model., 2020, 60, 6147-6154.

17 G. X. Sun, Y. D. Jin, S. Z. Li, Z. C. Yang, B. M. Shi, C. Chang and Y. A. Abramov, J. Phys. Chem. Lett., 2020, 11, 8832-8838.

18 A. R. Hill, P. Cubillas, J. T. Gebbie-Rayet, M. Trueman, N. de Bruyn, Z. al Harthi, R. J. S. Pooley, M. P. Attfield, V. A. Blatov, D. M. Proserpio, J. D. Gale, D. Akporiaye, B. Arstad and M. W. Anderson, Chem. Sci., 2021, 12, 1126-1146.

19 J. Nyman and S. M. Reutzel-Edens, Faraday Discuss., 2018, 211, 459-476.

20 C. X. Zhao, L. J. Chen, Y. Che, Z. F. Pang, X. F. Wu, Y. X. Lu, H. L. Liu, G. M. Day and A. I. Cooper, Nat. Commun., 2021, 12, 1-11.

21 N. Artrith, K. T. Butler, F. X. Coudert, S. Han, O. Isayev, A. Jain and A. Walsh, Nat. Chem., 2021, 13, 505-508.

22 J. J. Devogelaer, H. Meekes, P. Tinnemans, E. Vlieg and R. de Gelder, Angew. Chem., Int. Ed., 2020, 59, 21711-21718.

23 A. P. Frade, P. McCabe and R. I. Cooper, CrystEngComm, 2020, 22, 7186-7192.

24 A. Y. T. Wang, R. J. Murdock, S. K. Kauwe, A. O. Oliynyk, A. Gurlo, J. Brgoch, K. A. Persson and T. D. Sparks, Chem. Mater., 2020, 32, 4954-4965.

25 D. S. Hughes, M. B. Hursthouse, T. Threlfall and S. Tavener, Acta Crystallogr., Sect. C: Cryst. Struct. Commun., 1999, 55, 1831-1833.

26 M. B. Hursthouse, D. S. Hughes, T. Gelbrich and T. L. Threlfall, Chem. Cent. J., 2015, 9, 1.

27 T. Gelbrich, D. S. Hughes, M. B. Hursthouse and T. L. Threlfall, CrystEngComm, 2008, 10, 1328-1334.

28 A. L. Bingham, D. S. Hughes, M. B. Hursthouse, R. W. Lancaster, S. Tavener and T. L. Threlfall, Chem. Commun., 2001, 603-604.

29 B. T. Ibragimov, S. A. Talipov and P. M. Zorky, Supramol. Chem., 1994, 3, 147-165.

30 C. R. Groom, I. J. Bruno, M. P. Lightfoot and S. C. Ward, Acta Crystallogr., Sect. B: Struct. Sci., Cryst. Eng. Mater., 2016, 72, 171-179.

31 J. A. Chisholm and S. Motherwell, J. Appl. Crystallogr., 2005, 38, 228-231.

32 C. F. Macrae, I. Sovago, S. J. Cottrell, P. T. A. Galek, P. McCabe, E. Pidcock, M. Platings, G. P. Shields, J. S. Stevens, M. Towler and P. A. Wood, J. Appl. Crystallogr., 2020, 53, 226-235.

33 J. Rohlicek, E. Skorepova, M. Babor and J. Cejka, J. Appl. Crystallogr., 2016, 49, 2172-2183.

34 J. Rohlicek, E. Skorepova, M. Babor and J. Cejka, J. Appl. Crystallogr., 2016, 49, 2172-2183.

35 T. Gelbrich and M. B. Hursthouse, CrystEngComm, 2005, 7, 324-336.

36 C. C. Seaton, R. R. Thomas, E. A. A. Essifaow, E. Nauha, T. Munshi and I. J. Scowen, CrystEngComm, 2018, 20, 3428-3434. 
37 A. L. Spek, Acta Crystallogr., Sect. C: Struct. Chem., 2015, 71, 9-18.

38 D. C. Apperley, R. A. Fletton, R. K. Harris, R. W. Lancaster, S. Tavener and T. L. Threlfall, J. Pharm. Sci., 1999, 88, 1275-1280.

39 E. L. Eliel, S. H. Wilen and L. N. Mander, Stereochemistry of Organic Compounds, Wiley, New York, 1994.

40 J. van de Streek and M. A. Neumann, Acta Crystallogr., Sect. B: Struct. Sci., 2010, 66, 544-558.

41 A. Gavezzotti, New J. Chem., 2011, 35, 1360-1368.

42 I. Sovago, M. J. Gutmann, J. G. Hill, H. M. Senn, L. H. Thomas, C. C. Wilson and L. J. Farrugia, Cryst. Growth Des., 2014, 14, 1227-1239.

43 A. D. Bond, J. Appl. Crystallogr., 2014, 47, 1777-1780.

44 A. L. Spek, Acta Crystallogr., Sect. D: Biol. Crystallogr., 2009, 65, 148-155.
45 A. Kálmán, M. Czugler and G. Argay, Acta Crystallogr., Sect. B: Struct. Crystallogr. Cryst. Chem., 1981, 37, 868-877.

46 A. J. Cruz-Cabeza and J. Bernstein, Chem. Rev., 2014, 114, 2170-2191.

47 R. E. Rosenfield, R. Parthasarathy and J. D. Dunitz, J. Am. Chem. Soc., 1977, 99, 4860-4862.

48 X. J. Zhang, Z. Gong, J. Li and T. Lu, J. Chem. Inf. Model., 2015, 55, 2138-2153.

49 S. L. Childs, P. A. Wood, N. Rodriguez-Hornedo, L. S. Reddy and K. I. Hardcastle, Cryst. Growth Des., 2009, 9, 1869-1888.

50 T. Gelbrich and M. B. Hursthouse, CrystEngComm, 2006, 8, 448-460.

51 T. Gelbrich, M. B. Hursthouse and T. L. Threlfall, Acta Crystallogr., Sect. B: Struct. Sci., 2007, 63, 621-632. 